\title{
Enhanced External Counterpulsation Therapy: Significant Clinical Improvement without Electrophysiologic Remodeling
}

\author{
Charles A. Henrikson, M.D., and Nisha Chandra-Strobos, M.D. \\ From the Department of Medicine, Johns Hopkins Medical Institutions, Baltimore, Maryland
}

\begin{abstract}
Background: Enhanced external counterpulsation therapy (EECP), in addition to improving coronary flow and increasing the time to ischemia, noninvasively alters hemodynamics in patients with severe coronary artery disease (CAD). Other treatments that alter hemodynamics, for example, balloon valvuloplasty, left ventricular assist devices, and pharmacologic antagonism of the rennin-angiotensin system, promote electrophysiologic remodeling, as evidenced by alterations in the QT interval.

Methods: We studied 28 patients who completed a 7-week, 35-hour session of EECP to assess whether such therapy would also result in electrophysiologic remodeling.

Results: All patients had class II-III angina, imaging-proven ischemia, and severe, near-inoperable CAD. Of 28 patients, with a mean age $62 \pm 13$ years (mean $\pm \mathrm{SD}$ ), $78 \%$ were male, $46 \%$ diabetic, $82 \%$ hypertensive, $60 \%$ had undergone angioplasty, and $67 \%$ had undergone bypass surgery. The mean ejection fraction was 44\% (range 25-60\%). Following EECP, most patients (82\%) had at least a one full class improvement in their anginal pattern. In most patients, there was substantial baseline conduction system disease present: a mean QRS of $105 \pm 19 \mathrm{~ms}$. It is to be noted that there was no significant change in heart rate (HR), $\mathrm{PR}, \mathrm{QRS}$, or $\mathrm{QT}_{\mathrm{C}}$ intervals before and after EECP in either clinical responders or nonresponders. When analyzed by response to EECP, ejection fraction, or history of revascularization, there were still no detectable changes in ECG parameters (all P $=N S$ ).

Conclusions: While EECP remains an effective treatment for severe CAD, it does not prompt early electrical remodeling of the heart.

A.N.E. 2004;9(3):265-269
\end{abstract}

coronary artery disease; enhanced external counterpulsation; electrocardiogram; electrophysiology

Enhanced external counterpulsation therapy (EECP) noninvasively improves coronary flow and time to ischemia in patients with severe coronary artery disease (CAD). ${ }^{1,2}$ EECP acutely increases preload to the heart, and decreases afterload, both of which tend to lead to increased oxygen delivery to the myocardium during treatment. ${ }^{3}$ The mechanisms of action of EECP are under active investigation, and most studies have focused on collateral development ${ }^{4}$ or improvements in the endothelial function. ${ }^{5}$

The cardiac conduction system of the heart tends to become diseased with age, coronary artery disease, and heart failure. Increased QRS duration and increased QT interval are two powerful markers for the risk of sudden death in patients with heart failure and coronary artery disease. ${ }^{6}$ Primary angioplasty, ${ }^{7}$ angiotensin receptor blockers ${ }^{8}$ and converting enzyme inhibitors, ${ }^{9,10}$ balloon valvuloplasty, ${ }^{11,12}$ and left ventricular assist devices ${ }^{13}$ all cause alterations in QT interval. Each of these interventions causes a change in the loading conditions of the heart and the changes in QT interval are most likely the result of "contraction-excitation" feedback, that is, mechanical strain leading to changes in membrane potential and subsequent changes in repolarization. ${ }^{14}$

EECP causes major changes in hemodynamics, similar to those of the interventions described above. However, there have been no investigations of possible electrophysiologic effects of EECP. In analogy to other interventions that change hemodynamics and alter electrophysiologic parameters, we hypothesized that EECP could result in 
electrical remodeling of the cardiac conduction system as reflected by changes in the surface ECG, which may serve as a marker for clinical response, and may indicate a heretofore-unrecognized benefit of EECP.

\section{METHODS}

All patients had class II-III angina, imagingproven ischemia, or severe, near-inoperable CAD, and were referred for EECP by their primary care physicians or their cardiologists. EECP was performed in the conventional manner: ${ }^{15} 35$ one-hour sessions of therapy over 7 weeks in which pneumatic cuffs around the legs and lower abdomen are inflated and deflated during diastole at 0.4 pounds per square inch (psi) with the inflation/deflation signal being manually adjusted to maximize pressure augmentation as measured by finger plethysmography. The Johns Hopkins Bayview Medical Center Institutional Review Board granted approval of the study.

We examined the surface electrocardiograms of patients referred for EECP. The most recent ECG done prior to EECP (but within 6 weeks of initiation of therapy) was compared to an ECG done immediately following the completion of the therapy. Patients were excluded if they were in a paced rhythm, in atrial fibrillation or atrial flutter, if ECGs were unavailable, or if they did not complete EECP treatment. Standard clinical interpretation was applied to each ECG in the study, with RR, PR, QRS, and QT intervals having their usual meanings. The corrected QT interval was calculated using Bazett's $\operatorname{method}\left(\mathrm{QT}_{\mathrm{c}}=\mathrm{QT} / \sqrt{ } \mathrm{RR}\right)$.

Assessment of response to EECP therapy was determined by the cardiologist in charge of the EECP program, and by the patients' primary care physician. It was based on self-reported exercise tolerance, nitroglycerin use, and, if available, formal stress testing.

We used the $\mathrm{QT}_{\mathrm{c}}$ interval as the primary outcome variable and assumed a baseline mean $\mathrm{QT}_{\mathrm{c}}$ duration of $420 \mathrm{~ms}$ and a standard deviation of 25 ms. Assuming a significance level of $0.05,25$ patients would be required to have a power of $80 \%$ to detect a difference of $20 \mathrm{~ms}$ in $\mathrm{QT}_{\mathrm{c}}$ duration in the before and after measurements. Data are expressed as mean \pm standard deviation. Data were analyzed using the Stata 8 (Stata Corporation) software package. Before and after comparisons were made using paired $t$-tests.

\section{RESULTS}

We studied a total of 28 patients. Their baseline characteristics are given in Table 1 . All of the patients had advanced coronary artery disease, and most of them had prior myocardial infarction and coronary revascularization with percutaneous transluminal coronary angioplasty (PTCA) and/or coronary artery bypass grafting (CABG); 12 of 28 $(43 \%)$ patients had a depressed ejection fraction $(\leq 40 \%)$. Most of the patients had a meaningful clinical response to EECP ( $\geq 1$ class improvement in Canadian Cardiovascular Anginal Class). Five of 29 $(17 \%)$ had a lesser degree of improvement and were considered "nonresponders."

The summarized results of the surface ECGs are given in Figure 1. In most patients there was substantial baseline conduction system disease present: a mean QRS of $105 \pm 19 \mathrm{~ms}$. It is of note that there was no significant change in heart rate (HR), PR, QRS, or $\mathrm{QT}_{c}$ intervals before and after

Table 1. Patient Demographics and Baseline Characteristics $(\mathrm{n}=28)$

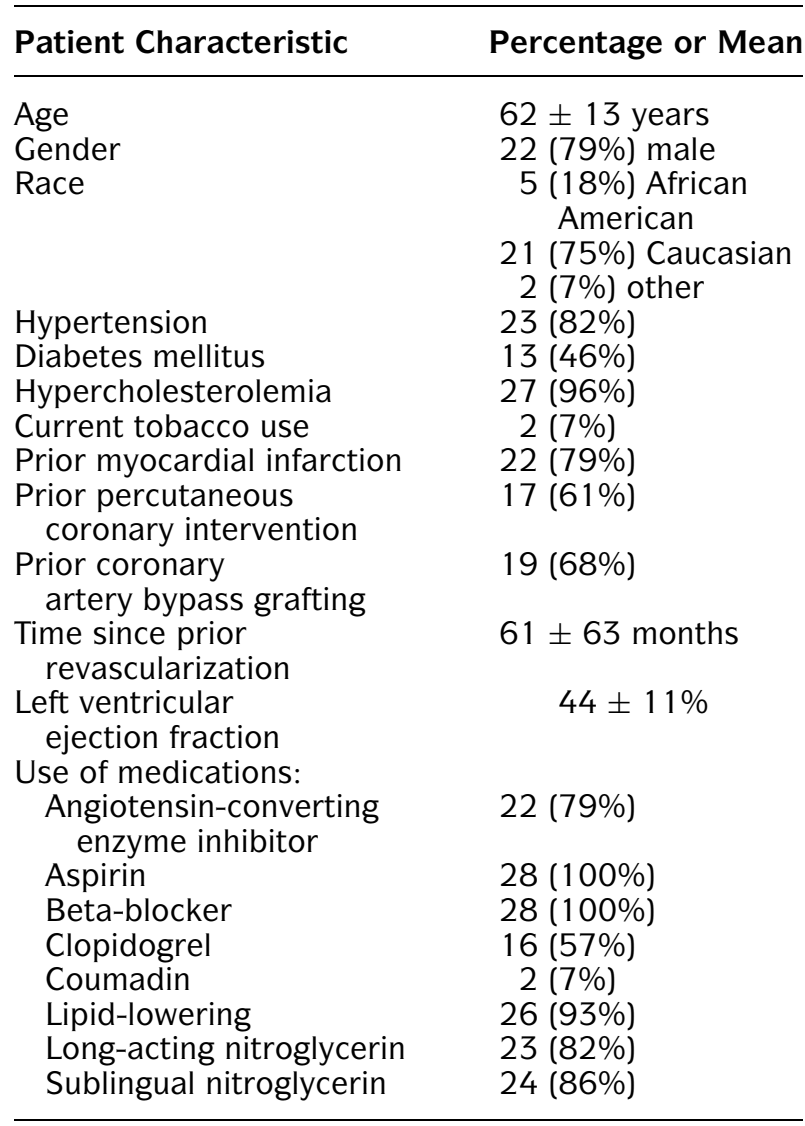




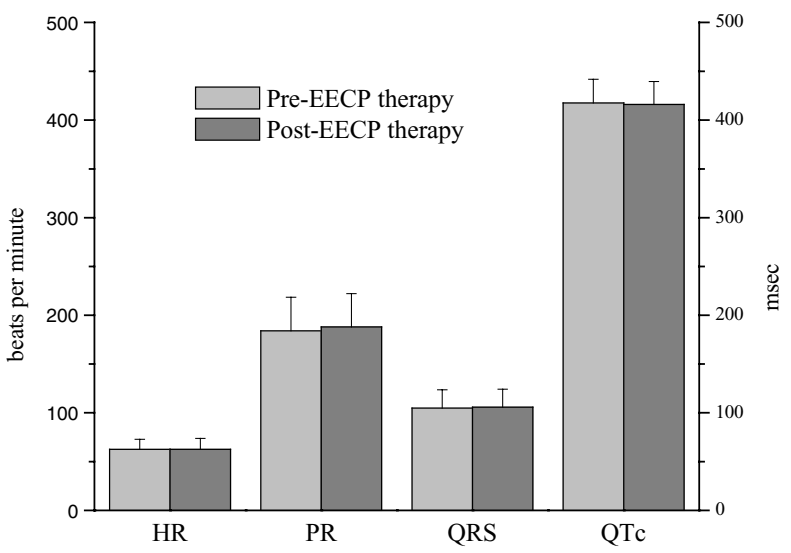

Figure 1. Electrocardiographic parameters pre- and post-external counterpulsation therapy. Data reported as mean \pm standard deviation. $H R=$ heart rate, and PR, $\mathrm{QRS}$, and $\mathrm{QT}_{\mathrm{c}}$ have their usual meanings derived from the surface electrocardiogram.

EECP in either clinical responders or nonresponders. In Figure 2, the individual changes in the $\mathrm{QT}_{\mathrm{c}}$ interval are displayed for each patient. When analyzed by response to EECP, ejection fraction, or history of revascularization, there were still no detectable changes in ECG parameters (all $\mathrm{P}=\mathrm{NS}$ ) (Table 2).

In spite of this lack of change of ECG parameters secondary to EECP, there were some differences observed in the subgroup analyses. As shown in Table 2, patients with lower left ventricular ejection fractions had a longer PR and a longer QRS

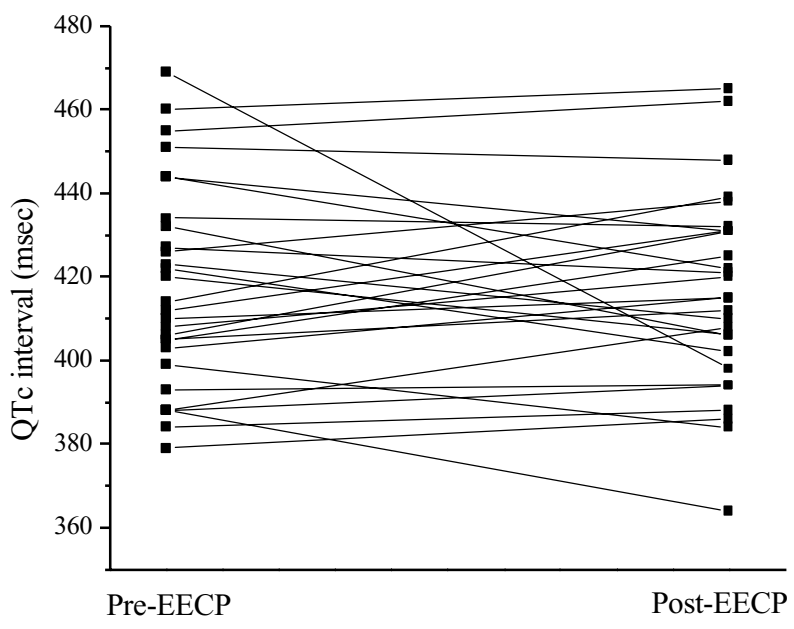

Figure 2. $\mathrm{QT}_{\mathrm{C}}$ values pre- and post-EECP. $\mathrm{QT}_{\mathrm{C}}$ intervals are plotted for each patient pre- and post-EECP. prior to EECP, and had a higher heart rate following EECP. Additionally, patients who did not have a robust response to EECP therapy had a higher heart rate following therapy than those who responded well.

\section{DISCUSSION}

EECP acutely increases coronary blood flow, improves symptoms of coronary artery disease, decreases nitroglycerin requirement, increases time to ST depression on stress tests, improves cardiac perfusion by nuclear imaging, and improves peripheral vascular endothelial reactivity. ${ }^{3,15-18}$ The acute hemodynamic effects of EECP are similar to those of intraaortic balloon counterpulsation. ${ }^{19}$ In spite of these data, the long-term mechanisms underlying the benefits of EECP have not been fully elucidated. ${ }^{2,5}$ An increase in wall sheer stress is known to improve endothelial function ${ }^{18}$ and EECP increases sheer wall stress, and this may contribute to some of EECP's acute beneficial effects in the heart and in the peripheral vasculature. However, sheer stress alone does not explain all of the benefits of EECP. ${ }^{4,5}$ Hence, several avenues of investigation are being pursued to better determine other mechanisms that may be etiologically responsible for the long-term clinical benefit noted in such patients. ${ }^{2,5}$

Severe coronary artery disease, with limited revascularization options, remains the only FDAand Medicare-approved indication for EECP. ${ }^{20}$ Such patients often have a disease of their native conduction system, as demonstrated by a widened QRS, QT interval prolongation, or various degrees of heart block, as is found in any population with severe CAD ${ }^{6}$ In general, this conduction system disease is felt to be the result of coronary artery disease: ischemia in the conduction system leads to remodeling and delayed conduction.

EECP causes acute changes in hemodynamics including an increase in preload and a decrease in afterload. ${ }^{3}$ There have been no studies of chronic changes in hemodynamics following EECP. However, the clinical improvement derived from EECP persists for up to 5 years. ${ }^{21}$ Other treatments that acutely alter hemodynamics have been shown to affect surface ECG parameters via the contractionexcitation feedback. ${ }^{14}$ Percutaneous valvuloplasty in children with congenital heart disease leads to a lengthening of the $\mathrm{QT}_{\mathrm{c}}$ interval for procedures involving either the aortic or pulmonic valves. ${ }^{11,12}$ Aortic procedures usually result in a decrease in 
268 • A.N.E. • July 2004 • Vol. 9, No. 3 • Henrikson and Chandra-Strobos • EECP and ECG

Table 2. Subgroup Analysis

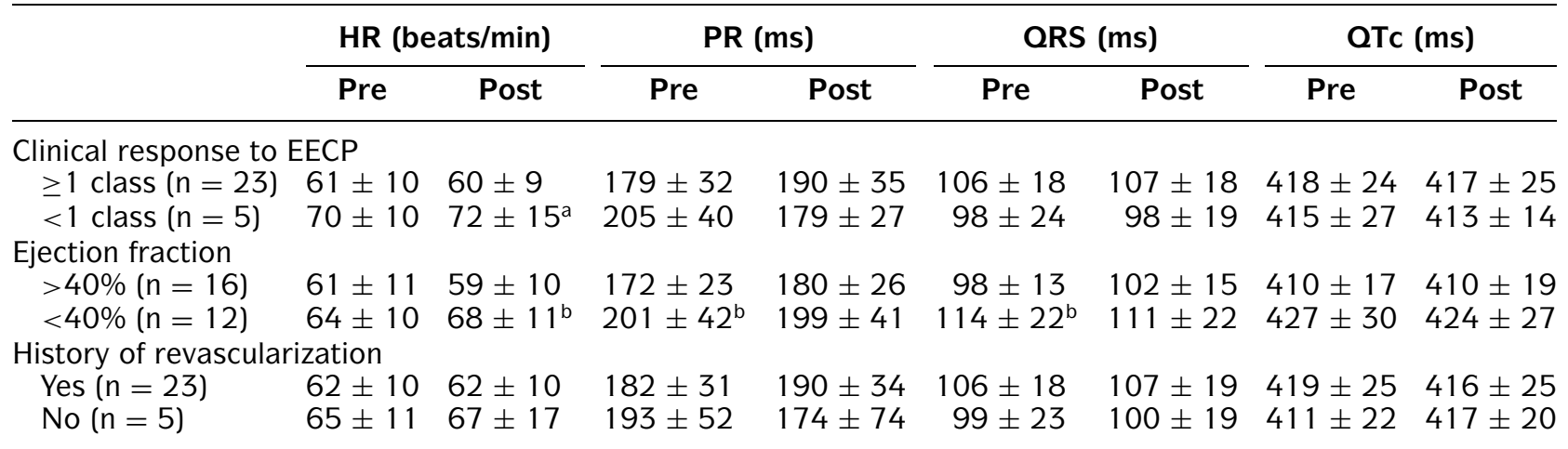

${ }^{a} \mathrm{P}<0.05$ for comparison with $\geq 1$ class improvement, ${ }^{\mathrm{b}} \mathrm{P}<0.05$ for comparison with $\mathrm{EF}>40 \%, \mathrm{P}>0.05$ for all other comparisons.

afterload, whereas pulmonic procedures have a more complicated hemodynamic effect. In several patients who underwent left ventricular assist device (LVAD) placement, Harding et al. ${ }^{13}$ found that the QRS narrowed and the QT initially lengthened and then narrowed. They attributed this effect to the massive unloading of the heart by the LVAD, and confirmed the changes by examined action potentials from the explanted hearts. Pharmaceuticals that block the renin-angiotensin system lead to a decrease in $\mathrm{QT}_{\mathrm{c}}$ over time. ${ }^{8-10}$ While these drugs have myriad effects, a reduction in afterload is prominent. However, other therapies that are suspected of having effects on the cardiac conduction system have been shown not to have any effects on surface ECG parameters. Both estrogen replacement therapy ${ }^{22}$ and n-3 fatty acid supplementation $^{23}$ do not have an effect on surface ECG parameters. Additionally, animal studies have shown that an increase in preload leads to a shortening of the action potential, which is analogous to a shortening of the QT interval. ${ }^{24}$

Given the myriad of interventions that can lead to both lengthening and shortening of the $\mathrm{QT}_{\mathrm{c}}$ interval, we undertook this study to determine if EECP would have any effect on the $\mathrm{QT}_{\mathrm{c}}$ interval. In the prior clinical studies detailed above, in general it appears that a decrease in afterload leads to a shortening of the $\mathrm{QT}_{\mathrm{c}}$ interval, while the effects of a change in preload are less dramatic. In dog studies, however, there was a marked decrease in the $\mathrm{QT}_{\mathrm{c}}$ interval with increased preload. Given the decrease in afterload, and the dramatic increase in preload demonstrated by Michaels et al. ${ }^{3}$ by 17 and $66 \mathrm{~mm}$ $\mathrm{Hg}$ respectively), we theorized that EECP may result in a change in the $\mathrm{QT}_{\mathrm{c}}$ interval, as well as other surface ECG parameters. We reasoned that EECP could result in "reverse" electrophysiologic remodeling of the heart, with a narrowing of the QRS, a shortening of the QT interval, and possibly a shortening of the PR interval. However, in this sample of 28 patients, our hypothesis was not substantiated.

Why did we not see changes in the $\mathrm{QT}_{\mathrm{c}}$ interval or other surface ECG parameters? First, our population consisted primarily of patients with coronary artery disease, rather than heart failure (although some of our patients did have (medication controlled) heart failure and a low ejection fraction). The possibility of remodeling to the degree that it is visible on the surface ECG may be easier to see if the hearts are extremely dilated and stressed, such as those in patients awaiting transplant, or with severe valvular heart disease, and the hemodynamic changes may be more pronounced in hearts that are failing.

The response to EECP varies from patient to patient, and the reasons for the variability in response to EECP remain a mystery. ${ }^{5,20}$ In this study, patients who had a robust response to EECP had a lower mean heart rate than did those who did not respond (Table 2). While all of the patients in the study were on beta-sympathetic antagonists, those with higher heart rates were most likely not as well beta-blocked as those with lower heart rates. The data presented here generate the hypothesis that adequate beta-blockade may be necessary for robust response to EECP.

While EECP does not seem to lead to electrophysiologic remodeling of the cardiac conduction system as reflected by the surface ECG, these data add to our understanding of the physiology of this novel therapy, and provide evidence that while EECP 
remains an effective treatment for severe $\mathrm{CAD}$, it does not prompt early electrical remodeling of the heart as reflected by the surface ECG.

\section{REFERENCES}

1. Beller GA. A review of enhanced external counterpulsation clinical trials. Clin Cardiol 2002;25:II6-II10.

2. Bonetti PO, Holmes DR, Lerman A, et al. Enhanced external counterpulsation for ischemic heart disease. What's behind the curtain? J Am Coll Cardiol 2003;41:1918-1925.

3. Michaels AD, Accad M, Ports TA, et al. Left ventricular systolic unloading and augmentation of intracoronary pressure and Doppler flow during enhanced external counterpulsation. Circulation 2002;106:1237-1242.

4. Cohn PF. EECP-New data on possible mechanisms of action. Eur Heart J 2001;22:1363-1364.

5. Feldman AM. Enhanced external counterpulsation: Mechanism of action. Clin Cardiol 2002;25:II11-II15.

6. Moss AJ, Fadl Y, Zareba W, et al. Survival benefit with an implanted defibrillator in relation to mortality risk in chronic coronary heart disease. Am J Cardiol 2001;88:516520.

7. Bonnemeier $\mathrm{H}$, Hartmann $F$, Wiegand UK, et al. Course and prognostic implications of QT interval and QT interval variability after primary coronary angioplasty in acute myocardial infarction. J Am Coll Cardiol 2001;37:44-50.

8. Oikarinen L, Nieminen MS, Toivonen L, et al. Relation of QT interval and QT dispersion to regression of echocardiographic and electrocardiographic left ventricular hypertrophy in hypertensive patients: the Losartan Intervention For Endpoint Reduction (LIFE) study. Am Heart J 2003;145:919-925.

9. Malmqvist $\mathrm{K}$, Kahan T, Edner M, et al. Comparison of actions of irbesartan versus atenolol on cardiac repolarization in hypertensive left ventricular hypertrophy: Results from the Swedish Irbesartan Left Ventricular Hypertrophy Investigation Versus Atenolol (SILVHIA). Am J Cardiol 2002;90:1107-1112.

10. Kassotis J, Mongwa M, Reddy CV. Effects of angiotensinconverting enzyme inhibitor therapy on QT dispersion post acute myocardial infarction. Pacing Clin Electrophysiol 2003;26:843-848

11. Levine $\mathrm{JH}$, Guarnieri $\mathrm{T}$, Kadish $\mathrm{AH}$, et al. Changes in myocardial repolarization in patients undergoing balloon valvuloplasty for congenital pulmonary stenosis: evidence for contraction-excitation feedback in humans. Circulation $1988 ; 77: 70-77$.
12. Martin GR, Stanger P. Transient prolongation of the QTc interval after balloon valvuloplasty and angioplasty in children. Am J Cardiol 1986;58:1233-1235.

13. Harding JD, Piacentino V III, Gaughan JP, et al. Electrophysiological alterations after mechanical circulatory support in patients with advanced cardiac failure. Circulation 2001; 104:1241-1247.

14. Lab MJ. Contraction-excitation feedback in myocardium. Physiological basis and clinical relevance. Circ Res 1982;50:757-766.

15. Arora RR, Chou TM, Jain D, et al. The multicenter study of enhanced external counterpulsation (MUST-EECP): Effect of EECP on exercise-induced myocardial ischemia and anginal episodes. J Am Coll Cardiol 1999;33:18331840.

16. Urano $H$, Ikeda $H$, Ueno $T$, et al. Enhanced external counterpulsation improves exercise tolerance, reduces exerciseinduced myocardial ischemia and improves left ventricular diastolic filling in patients with coronary artery disease. J Am Coll Cardiol 2001;37:93-99.

17. Stys TP, Lawson WE, Hui JC, et al. Effects of enhanced external counterpulsation on stress radionuclide coronary perfusion and exercise capacity in chronic stable angina pectoris. Am J Cardiol 2002;89:822-824.

18. Bonetti PO, Barsness GW, Keelan PC, et al. Enhanced external counterpulsation improves endothelial function in patients with symptomatic coronary artery disease. J Am Coll Cardiol 2003;41:1761-1768.

19. Taguchi I, Ogawa $K$, Oida A, et al. Comparison of hemodynamic effects of enhanced external counterpulsation and intraaortic balloon pumping in patients with acute myocardial infarction. Am J Cardiol 2000;86:11391141, A9.

20. Lawson WE. Current use of enhanced external counterpulsation and patient selection. Clin Cardiol 2002;25:II16-II21.

21. Lawson WE, Hui JC, Cohn PF. Long-term prognosis of patients with angina treated with enhanced external counterpulsation: five-year follow-up study. Clin Cardiol 2000;23:254-258

22. Vrtovec B, Starc V, Meden-Vrtovec H. The effect of estrogen replacement therapy on ventricular repolarization dynamics in healthy postmenopausal women. J Electrocardiol 2001;34:277-283

23. Geelen A, Brouwer IA, Zock PL, et al. (N-3) fatty acids do not affect electrocardiographic characteristics of healthy men and women. J Nutr 2002;132:3051-3054.

24. Lerman BB, Burkhoff D, Yue DT, et al. Mechanoelectrical feedback: Independent role of preload and contractility in modulation of canine ventricular excitability. J Clin Invest 1985;76:1843-1850. 